\title{
Dehn surgeries that yield fibred 3-manifolds
}

\author{
$\mathrm{Yi} \mathrm{Ni}$ \\ American Institute of Mathematics \\ 360 Portage Ave, Palo Alto, CA 94306-2244 \\ and \\ Department of Mathematics, Columbia University \\ MC 4406, 2990 Broadway, New York, NY 10027
}

February 2, 2008

\begin{abstract}
We study Dehn surgeries on null-homotopic knots that yield fibred 3-manifolds when an additional (but natural) homological restriction is imposed. The major tool used is Gabai's theory of sutured manifold decomposition. Such surgeries are negative examples to a question of Michel Boileau. Another result we will prove is about surgeries which reduce the Thurston norm of a fibred manifold.
\end{abstract}

\section{Introduction}

One basic question on Dehn surgery is when a Dehn surgery yields a special type of manifolds. In this paper, we will consider Dehn surgeries on nullhomotopic knots that yield fibred manifolds. All manifolds we study here will be compact and orientable unless stated otherwise. Our main result is as follows.

Theorem 1.1. Suppose $Y$ is a compact 3-manifold with boundary consisting of tori, $L$ is a nontrivial null-homotopic knot in $Y$. Let $\alpha$ be a nontrivial slope on $T=\partial \operatorname{Nd}(L), X$ is the manifold obtained from $Y$ by $\alpha$-surgery, $K \subset X$ is the core of the surgery.

If $X$ fibres over the circle with fibre $F$, such that

$$
[K] \cdot[F]=0,
$$

then there is an ambient isotopy of $X$ which takes $K$ to a curve in $F$. Moreover, let $\beta \subset T$ be the meridian of $L$, then $\beta$ is the frame of $K$ specified by $F \supset K$. Hence $\Delta(\alpha, \beta)=1$, where $\Delta$ is the distance between two slopes.

Corollary 1.2. Suppose $L$ is a nontrivial null-homotopic knot in a closed 3manifold $Y, X$ is obtained by a $\frac{p}{q}$-surgery on $L, \frac{p}{q} \neq 0, \infty$. If $X$ fibres over the circle, then $\frac{p}{q} \in \mathbb{Z}$, and every integer surgery on $L$ yields a fibred manifold. 
Proof. Let $K \in X$ be the core of the surgery. Since $\frac{p}{q} \neq 0, K$ is rationally null-homologous in $X$, hence the condition (1) is satisfied. Let $\alpha, \beta$ be as in Theorem 1.1, then $\Delta(\alpha, \beta)=1$, which implies that $\frac{p}{q} \in \mathbb{Z}$. Since $\beta$ is the frame of $K$ specified by $F$, every integer surgery on $L$ yields a fibred manifold whose monodromy differs from the monodromy of $X$ by a power of the Dehn twist along $K \subset F$.

Our theorem is related to the following question of Michel Boileau.

Question 1.3. [9, Problem 1.80C, Boileau] Let $L$ be a null-homotopic knot in a closed orientable $3-$ manifold $Y$. If a nontrivial surgery on $L$ yields a manifold that fibres over the circle, does it follow that $L$ is a fibred knot and the surgery is longitudinal?

This question was answered affirmatively in the case that $Y=S^{3}$ by Gabai 4. Boileau and Wang showed that if the surgery is fibred then either the surgery is longitudinal or $Y$ itself is fibred [1]. In [14] the question was answered affirmatively for null-homologous knots of genus $>1$ in $L$-spaces, using Heegaard Floer homology.

However, we note that there are simple examples of surgeries on null-homotopic knots yielding fibred manifolds and satisfying the homological restriction (11), hence they are negative examples to Question 1.3. A construction of such examples can be given as follows. Take any unknotting number one fibred knot $k \subset S^{3}$. There exists a circle $\gamma \subset S^{3}-k$ which has linking number zero with $k$ such that a \pm 1 surgery on $\gamma$ yields a solid torus, which means that there exists a winding number zero knot $L$ in the solid torus $U$, such that a surgery on $L$ yields the fibred manifold $S^{3}-k$. In order to construct knots in closed manifolds, one can take any closed manifold $Y$ which fibres over the circle, embed $U$ into $Y$ such that the core of $U$ is transverse to the fibres, then $L \subset Y$ is a knot that satisfies the conditions in Theorem 1.1.

The proof of Theorem 1.1 uses Gabai's theory of taut foliations and sutured manifold decomposition. Sutured manifold theory has been successfully applied to study surgery on null-homotopic knots by Lackenby [12]. In addition to the use of [3] as in [11, 12] we borrow some ideas from [4] and [6], which have been used to show that knot Floer homology detects fibred knots [6, 14].

The same argument can be used to prove the following theorem.

Theorem 1.4. Suppose $M$ is a compact 3-manifold with boundary consisting of tori, $T$ is a component of $\partial M, \alpha, \beta$ are two different slopes on $T, X, Y$ are the manifolds obtained by filling $T$ along $\alpha, \beta, K \subset X$ is the core of the $\alpha$-filling. Let $i_{X}: \mathrm{H}_{2}(M, \partial M-T) \rightarrow \mathrm{H}_{2}(X, \partial X), i_{Y}: \mathrm{H}_{2}(M, \partial M-T) \rightarrow \mathrm{H}_{2}(Y, \partial Y)$ be the maps on homology induced by inclusions.

Suppose $X$ fibres over the circle with fibre $F$ such that there exists a $\theta \in$ $\mathrm{H}_{2}(M, \partial M-T)$ satisfying $i_{X}(\theta)=[F]$. If the Thurston norm of $i_{Y}(\theta)$ is less than the Thurston norm of $F$, then there is an ambient isotopy of $X$ which takes $K$ to a curve in $F$. Moreover, $\beta$ coincides with the frame on $K$ which is specified by the surface $F$. Hence $\Delta(\alpha, \beta)=1$. 
Remark 1.5. The conclusion $\Delta(\alpha, \beta)=1$ in the above theorems can also be proved by the argument in [11, Theorem 5.2]. The conclusion of Theorem 1.4 has also been obtained by John Luecke [13] in the case when $Y$ is a solid torus. Our argument in this paper is closer to Lackenby's, while Luecke's argument involves the combinatorial techniques from [7].

Remark 1.6. In Theorem 1.4 it is interesting to ask what happens when $X$ is not fibred. One may naturally guess that $K$ can be isotoped to a curve in some taut surface representing $i_{X}(\theta)$, but this picture is not correct: Gabai told the author a method of constructing negative examples. In spite of this disappointing answer, the above guess is true in the special case when $X$ and $Y$

are link complements related by a crossing change, according to a result due to Scharlemann and Thompson [16, Proposition 3.1].

This work is motivated by works in Heegaard Floer homology [6, 14, but the argument here is quite classical, we do not need Heegaard Floer homology and contact topology at all. The only gauge theoretical aspect in this paper is the citation of Property P [10, which can be replaced by the Knot Complement Theorem [7] if we assume there are no fake $3-$ cells in $Y$. One should even be able to eliminate foliations as in [15, 11, 12.

The paper is organized as follows. In Section 2 we will give some preliminaries on sutured manifold decompositions. In Section 3 we study knots in product manifolds via a method of Gabai 4. The key result in this section is Proposition 3.4. The proofs of the above two theorems are just its routine applications, which are given in Section 4 and 5.

Acknowledgements. We are grateful to Michel Boileau, David Gabai and Tao Li for some very interesting discussions, to Marc Lackenby for helpful comments, and to John Luecke for a detailed description of his earlier proof of a special case of Theorem 1.4. The author is partially supported by an AIM Five-Year Fellowship. This research was partially conducted during the period the author was employed by the Clay Mathematics Institute as a Liftoff Fellow, and when the author visited University of Minnesota, Twin Cities. The author wishes to thank the above institutions for their supports, and special thanks are due to Tian-Jun Li for his hospitality.

\section{Preliminaries on sutured manifolds}

Sutured manifold decomposition was introduced by Gabai in 2] in order to construct taut foliations. In this section, we will briefly review some basic definitions about sutured manifolds, then discuss the main result in $[3]$.

Definition 2.1. A sutured manifold $(M, \gamma)$ is a compact oriented 3-manifold $M$ together with a set $\gamma \subset \partial M$ of pairwise disjoint annuli $A(\gamma)$ and tori $T(\gamma)$. The core of each component of $A(\gamma)$ is a suture, and the set of sutures is denoted by $s(\gamma)$. 
Every component of $R(\gamma)=\partial M-\operatorname{int}(\gamma)$ is oriented. Define $R_{+}(\gamma)$ (or $R_{-}(\gamma)$ ) to be the union of those components of $R(\gamma)$ whose normal vectors point out of (or into) $M$. The orientations on $R(\gamma)$ must be coherent with respect to $s(\gamma)$, hence every component of $A(\gamma)$ lies between a component of $R_{+}(\gamma)$ and a component of $R_{-}(\gamma)$.

Definition 2.2. Let $S$ be a compact oriented surface with connected components $S_{1}, \ldots, S_{n}$. We define

$$
x(S)=\sum_{i} \max \left\{0,-\chi\left(S_{i}\right)\right\} .
$$

Let $M$ be a compact oriented 3-manifold, $A$ be a compact codimension- 0 submanifold of $\partial M$. Let $h \in \mathrm{H}_{2}(M, A)$. The Thurston norm $x(h)$ of $h$ is defined to be the minimal value of $x(S)$, where $S$ runs over all the properly embedded surfaces in $M$ with $\partial S \subset A$ and $[S]=h$.

Definition 2.3. A properly embedded surface $S \subset M$ is taut, if $S$ is incompressible and Thurston norm minimizing in $\mathrm{H}_{2}(M, \partial S)$. A sutured manifold $(M, \gamma)$ is taut, if $M$ is irreducible, and $R(\gamma)$ is taut.

Definition 2.4. Let $(M, \gamma)$ be a sutured manifold, and $S$ a properly embedded surface in M, such that no component of $\partial S$ bounds a disk in $R(\gamma)$ and no component of $S$ is a disk with boundary in $R(\gamma)$. Suppose that for every component $\lambda$ of $S \cap \gamma$, one of 1) -3 ) holds:

1) $\lambda$ is a properly embedded non-separating arc in $\gamma$.

2) $\lambda$ is a simple closed curve in an annular component $A$ of $\gamma$ in the same homology class as $A \cap s(\gamma)$.

3) $\lambda$ is a homotopically nontrivial curve in a toral component $T$ of $\gamma$, and if $\delta$ is another component of $T \cap S$, then $\lambda$ and $\delta$ represent the same homology class in $\mathrm{H}_{1}(T)$.

Then $S$ is called a decomposition surface, and $S$ defines a sutured manifold decomposition

$$
(M, \gamma) \stackrel{S}{\rightsquigarrow}\left(M^{\prime}, \gamma^{\prime}\right),
$$

where $M^{\prime}=M-\operatorname{int}(\operatorname{Nd}(S))$ and

$$
\begin{aligned}
\gamma^{\prime} & =\left(\gamma \cap M^{\prime}\right) \cup \operatorname{Nd}\left(S_{+}^{\prime} \cap R_{-}(\gamma)\right) \cup \operatorname{Nd}\left(S_{-}^{\prime} \cap R_{+}(\gamma)\right), \\
R_{+}\left(\gamma^{\prime}\right) & =\left(\left(R_{+}(\gamma) \cap M^{\prime}\right) \cup S_{+}^{\prime}\right)-\operatorname{int}\left(\gamma^{\prime}\right), \\
R_{-}\left(\gamma^{\prime}\right) & =\left(\left(R_{-}(\gamma) \cap M^{\prime}\right) \cup S_{-}^{\prime}\right)-\operatorname{int}\left(\gamma^{\prime}\right),
\end{aligned}
$$

where $S_{+}^{\prime}\left(S_{-}^{\prime}\right)$ is that component of $\partial \mathrm{Nd}(S) \cap M^{\prime}$ whose normal vector points out of (into) $M^{\prime}$.

Definition 2.5. A decomposition surface is called a product disk, if it is a disk which intersects $s(\gamma)$ in exactly two points. A decomposition surface is called a product annulus, if it is an annulus with one boundary component in $R_{+}(\gamma)$, and the other boundary component in $R_{-}(\gamma)$. 
Definition 2.6. An I-cobordism between closed connected surfaces $T_{0}$ and $T_{1}$ is a compact 3-manifold $V$ such that $\partial V=T_{0} \cup T_{1}$ and for $i=0,1$ the induced maps $j_{i}: \mathrm{H}_{1}\left(T_{i}\right) \rightarrow \mathrm{H}_{1}(V)$ are injective.

It is noted in [3, Lemma 1.5] that for an I-cobordism $V$ between $T_{0}$ and $T_{1}$, the maps $j_{1}, j_{2}$ induce isomorphisms on $\mathrm{H}_{1}(\cdot ; \mathbb{Q})$. One then easily sees that $V$ has the same rational homology type as $T_{0} \times I$.

Definition 2.7. Let $M$ be a compact 3 -manifold, $S$ a properly embedded surface in $M$, and $T$ a toral component of $\partial M$ such that $T \cap S=\emptyset . M$ is $S_{T^{-}}$ atoroidal if boundary parallel tori are the only surfaces which are I-cobordant to $T$ by cobordisms contained in $M-S$. If the boundary component $T$ is understood, then we say that $M$ is $S$-atoroidal.

The main result in 3 is as follows.

Theorem 2.8 (Gabai). Let $M$ be a compact irreducible 3-manifold whose boundary consists of tori. $T$ is a component of $\partial M$ and $S$ is a taut surface representing a nontrivial element in $\mathrm{H}_{2}(M, \partial M-T), S \cap T=\emptyset$. If $M$ is $S_{T^{-}}$ atoroidal, then except at most one slope the manifold $N$ obtained by filling $M$ along a slope in $T$ possesses a taut foliation $\mathscr{F}$ such that $S$ is a compact leaf of $\mathscr{F}$, and the core $C$ of the filling is transverse to $\mathscr{F}$, hence $C$ is of infinite order in $\pi_{1}(N)$.

Sketch of proof. There exists a sequence

$$
(M, \partial M)=\left(M_{0}, \gamma_{0}\right) \stackrel{S=S_{1}}{\rightsquigarrow}\left(M_{1}, \gamma_{1}\right) \stackrel{S_{2}}{\rightsquigarrow} \ldots \stackrel{S_{n}}{\rightsquigarrow}\left(M_{n}, \gamma_{n}\right)
$$

of sutured manifold decompositions with the following properties:

1) Each $\left(M_{i}, \gamma_{i}\right)$ is taut and each separating component of $S_{i+1}$ is a product disk. T.)

2) All $S_{i}$ 's are disjoint from $T$. (Hence some component of $\gamma_{n}$ is the torus

3) $\left(M_{n}, \gamma_{n}\right)$ is a union of a product sutured manifold and a sutured manifold $(H, \delta)$, where $H=T^{2} \times I, T=T^{2} \times 0, \delta \cap\left(T^{2} \times 1\right) \neq \emptyset$.

The idea is to inductively construct sutured manifold decompositions satisfying 1) and 2) until such construction can no longer be done. Now the last sutured manifold $\left(M_{n}, \gamma_{n}\right)$ should be a union of a product sutured manifold and a sutured manifold $(H, \delta)$, where $H$ is an I-cobordism between $T$ and another torus $P \subset M-S$. Since $M$ is $S_{T^{-}}$atoroidal, $H$ must be $T^{2} \times I$.

Fix a slope on $T$, we fill each $T \subset \partial M_{i}$ along this slope by a solid torus, to get the sequence

$$
\mathcal{N}: \quad(N, \partial M-T)=\left(N_{0}, \delta_{0}\right) \stackrel{S_{1}}{\rightsquigarrow}\left(N_{1}, \delta_{1}\right) \stackrel{S_{2}}{\rightsquigarrow} \ldots \stackrel{S_{n}}{\rightsquigarrow}\left(N_{n}, \delta_{n}\right) .
$$

By 3), the component $\widehat{H}$ of $N_{n}$ containing $T$ satisfies $\widehat{H}=D^{2} \times S^{1}$ and $s\left(\delta_{n}\right) \cap \widehat{H}$ is a union of $2 r(\neq 0)$ parallel essential simple closed curves in $\partial D^{2} \times S^{1}$. If the slope on $T$ is not the one that kills $s\left(\delta_{n}\right) \cap \widehat{H}$ in $\pi_{1}(\widehat{H})$, then one can decompose 
$\left(N_{n}, \delta_{n}\right)$ along a $D^{2} \times$ point to get a product sutured manifold. Hence the above sequence $\mathcal{N}$ is extended to a sutured manifold hierarchy.

Now we can apply [2, Theorem 5.1] to the sutured manifold hierarchy gotten in the last paragraph to obtain the desired foliations.

\section{Knots in product manifolds}

In this section we will study knots in product manifolds. Let $F$ be a compact surface, $K \subset F \times I$ is a knot which is not contained in a 3 -ball, (hence $F \neq$ $D^{2}$ or $\left.S^{2},\right) M_{1}=F \times I-\operatorname{int}(\operatorname{Nd}(K)), T=\partial N d(K)$.

Lemma 3.1. Suppose $R \subset M_{1}$ is a torus which is I-cobordant to T, then $R$ bounds a solid torus $U$ in $F \times I, K \subset U$.

Proof. Let $V$ be the I-cobordism between $T$ and $R$. If $R$ is incompressible in $F \times I$, then $R$ is isotopic to $F \times t$, which is impossible since $[R]=0$ in $\mathrm{H}_{2}(F \times I)$.

Now $R$ is compressible in $F \times I$, let $S$ be the sphere obtained by compressing $R$, then $R$ is obtained by adding a tube to $S$. $S$ bounds a ball $B$ in $F \times I$.

If the tube is contained in $B$, then $R$ bounds a cube-with-knotted-hole $U \subset B$ in $F \times I . K \subset F \times I-U$ since $K \not \subset B$. Now $M_{1}=U \cup V$ has only one boundary component $T$, which contradicts to the fact that $M_{1}=F \times I-\operatorname{int}(\operatorname{Nd}(K))$.

If the tube is not contained in $B$, then $R$ bounds a solid torus $U$. The same argument as in the last paragraph shows $K \subset U$.

Definition 3.2. Suppose $E$ is a compact subsurface of a compact surface $F$. $E$ is essential if no component of $\operatorname{Fr}(E)=E \cap \overline{F-E}$ is a circle that bounds a disk in $F$ or an proper arc that cobounds a disk in $F$ with an arc in $\partial F$.

Let $E \times I \subset M_{1}$ be the characteristic product pair of $M_{1}$. Namely, $E$ is a maximal (up to isotopy) compact essential subsurface of $F$, such that $K$ can be isotoped in $F \times I$ to be disjoint from $E \times I$. Let $M_{2}=\overline{M_{1}-E \times I}, G=\overline{F-E}$. Now $K$ is a knot in $G \times I$. By the choice of $E$, its complement $G$ should be connected. Let $\gamma_{1}=(\partial F \times I) \cup T, \gamma_{2}=(\partial G \times I) \cup T$, then $\left(M_{1}, \gamma_{1}\right),\left(M_{2}, \gamma_{2}\right)$ are sutured manifolds.

Definition 3.3. Suppose $S \subset M_{2}$ is a non-separating decomposition surface which gives a taut decomposition of $\left(M_{2}, \gamma_{2}\right), S \cap T=\emptyset . S$ is tautly extendable if $S$ also gives a taut decomposition of $(G \times I, \partial G \times I)$. $M_{2}$ has the taut-extension property, if every non-separating decomposition surface $S \subset M_{2}-T$ which gives a taut decomposition of $\left(M_{2}, \gamma_{2}\right)$ is tautly extendable.

Proposition 3.4. Suppose $M_{2}$ has the taut-extension property. The inclusion $K \subset G \times I$ induces a map

$$
i_{*}: \mathrm{H}_{1}(K ; \mathbb{Q}) \rightarrow \mathrm{H}_{1}(G ; \mathbb{Q}) .
$$

If $\sigma$ is a nonzero element in $\mathrm{H}_{1}(G, \partial G ; \mathbb{Q})$, then $\sigma \cdot i_{*}([K]) \neq 0$. 
Otherwise there exists a non-separating simple oriented curve (which is a circle or a proper arc) $C \subset G$ such that $[C] \cdot i_{*}([K])=0$.

Case 1. The curve $C$ is a proper arc with ends on different components of $\partial G$.

Suppose $\sigma, \tau$ are the two components of $\partial G$ that contain $\partial C, a \in \sigma-\partial C$ is a point. For a proper surface $S \subset M_{2}$, let $\partial_{i}(S)=S \cap(G \times i), i=0,1$, $\partial_{v}(S)=S \cap(\partial G \times I)$.

Let $\mathcal{S}_{m}(+C)$ be the set of properly embedded oriented surfaces $S \subset G \times I$, such that $S \cap K=\emptyset, \partial_{0} S=C \times 0, \partial_{1} S=-C \times 1$, and the algebraic intersection number between $S$ and $a \times I$ is $m$. Here $-C$ denotes the same curve $C$, but with opposite orientation. Similarly, let $\mathcal{S}_{m}(-C)$ be the set of properly embedded surfaces $S \subset G \times I$, such that $S \cap K=\emptyset, \partial_{0} S=-C \times 0, \partial_{1} S=C \times 1$, and the algebraic intersection number of $S$ with $a \times I$ is $m$. Since $[C] \cdot i_{*}([K])=0$, $\mathcal{S}_{m}( \pm C) \neq \emptyset$.

Lemma 3.5. When $m$ is sufficiently large, there exists a connected surface $S \subset \mathcal{S}_{m}(+C)$ such that $S$ gives a taut decomposition of $M_{2}$. The same statement holds for $S_{m}(-C)$.

This lemma is implicitly contained in [2, Theorem 3.13], and the details are given in [14, Lemma 6.4].

Suppose $S \subset M_{2}$ is a properly embedded surface which is transverse to $\partial G \times 0$. For any component $S_{0}$ of $S$, we define

$$
y\left(S_{0}\right)=\max \left\{\frac{\left|S_{0} \cap(\partial G \times 0)\right|}{2}-\chi(G), 0\right\},
$$

and let $y(S)$ be the sum of $y\left(S_{0}\right)$ with $S_{0}$ running over all components of $S$. Let $y\left(\mathcal{S}_{m}( \pm C)\right)$ be the minimal value of $y(S)$ for all $S \in \mathcal{S}_{m}( \pm C)$. If $S \in \mathcal{S}_{m}( \pm C)$, let $S^{\prime}$ be the surface obtained by doing oriented cut-and-paste to $S$ and $G \times 1$, it is obvious that $S^{\prime} \in \mathcal{S}_{m+1}( \pm C)$ and $y\left(S^{\prime}\right)=y(S)+y(G)$. Hence we have

$$
y\left(\mathcal{S}_{m+1}( \pm C)\right) \leq y\left(\mathcal{S}_{m}( \pm C)\right)+y(G) .
$$

The following key lemma is essentially [14, Lemma 6.5], the argument in the proof is due to Gabai 4].

Lemma 3.6. For any positive integers $p, q$,

$$
y\left(\mathcal{S}_{p}(+C)\right)+y\left(\mathcal{S}_{q}(-C)\right)>(p+q) y(G) .
$$

Suppose $S_{1} \in \mathcal{S}_{p}(+C), S_{2} \in \mathcal{S}_{q}(-C), p, q>0$, and $y\left(S_{1}\right)=y\left(\mathcal{S}_{p}(+C)\right)$, $y\left(S_{2}\right)=y\left(\mathcal{S}_{q}(-C)\right)$. Isotope $S_{1}, S_{2}$ so that they are transverse, and $\mid\left(\partial_{v} S_{1}\right) \cap$ $\left(\partial_{v} S_{2}\right) \mid$ is minimal. The following lemma is obvious.

Lemma 3.7. On $\sigma \times I, \partial_{v} S_{1}$ and $\partial_{v} S_{2}$ have exactly $p+q+1$ intersection points, and their orientations are the same. The same statement holds for $\tau \times I$.

Now $S_{1} \cap S_{2}$ consists of some circles and exactly $p+q+1$ arcs, each arc has one end on $\sigma \times I$ and the other end on $\tau \times I$. Note that two arcs among them are $C \times\{0,1\}$. 
Perform oriented cut-and-paste to $S_{1}, S_{2}$, we get a proper surface $P$, then we isotope $P$ slightly such that it lies $\operatorname{in} \operatorname{int}\left(M_{2}\right)$. It is easy to show that $\chi(P)=\chi\left(S_{1}\right)+\chi\left(S_{2}\right)-2$, hence

$$
y(P)=y\left(S_{1}\right)+y\left(S_{2}\right) .
$$

Definition 3.8. A properly embedded surface in $M_{2}$ is boring, if its Euler characteristic is nonnegative, and its algebraic intersection number with $a \times I$ is 0 .

Claim 0. There is exactly one component of $S_{1}$ whose intersection with $G \times$ $\{0,1\}$ is nonempty. Moreover, this component is not a disk or annulus.

Since $S_{1} \cap(G \times\{0,1\})=C \times\{0,1\}$, the component of $S_{1}$ which contain $C \times 0$ must also contain $C \times 1$. The second statement holds since $M_{2}$ contains no nontrivial product disks or product annuli.

Claim 1. We can assume that no component of $\partial S_{1}, \partial S_{2}$ is the boundary of a disk in $\partial G \times I$. Moreover, we can assume that $S_{1}, S_{2}$ contain no boring components.

If one component of $\partial S_{1}$ is the boundary of a disk in $\partial G \times I$, without loss of generality we can assume no other components of $\partial S_{1}, \partial S_{2}$ are contained in the disk, then we can cap off this component of $\partial S_{1}$ by the disk to get a new surface $S_{1}^{\prime} \subset \mathcal{S}_{p}(+C), y\left(S_{1}^{\prime}\right) \leq y\left(S_{1}\right)$. This proves the first statement.

Suppose $B$ is a boring component of $S_{1}$. We can remove $B$ without increasing $y\left(S_{1}\right)$, and the new surface is still contained in $\mathcal{S}_{p}(+C)$.

Claim 2. We can assume that no component of $S_{1} \cap S_{2}$ bounds a disk in $S_{1}$ or $S_{2}$, hence no component of $P$ is a boring sphere or disk.

If a component of $S_{1} \cap S_{2}$ bounds a disk in $S_{1}$, then this component also bounds a disk in $S_{2}$ since $S_{2}$ is incompressible. Since $M_{2}$ is irreducible, we can isotope $S_{1}$ to eliminate the components of $S_{1} \cap S_{2}$ that bound disks in $S_{1}$ or $S_{2}$. If a component $Q$ of $P$ is a boring sphere or disk, then $Q$ is a component of $S_{1}$ or $S_{2}$, since $S_{1} \cap S_{2}$ contains no circle that bounds a disk in $S_{1}$ or $S_{2}$. Now we apply Claim 1 to get a contradiction.

Claim 3. We can assume that there is no subsurface $Q$ of $P$, such that $Q$ is the union of some components of $P, Q \cdot(a \times I)=0$, and $\chi(Q)=0$.

Suppose $Q$ is such a subsurface of $P$, by Claims 1 and $2 Q$ is the union of two collections of annuli or tori $A_{1}, A_{2}, \ldots, A_{m}$ and $B_{1}, \ldots, B_{n}$, where $A_{i} \subset S_{1}$, $B_{j} \subset S_{2}$. Let

$$
\begin{aligned}
& S_{1}^{\prime}=\left(S_{1}-\bigcup_{i=1}^{m} A_{i}\right) \cup \bigcup_{j=1}^{n}\left(-B_{j}\right) \\
& S_{2}^{\prime}=\left(S_{2}-\bigcup_{j=1}^{n} B_{j}\right) \cup \bigcup_{i=1}^{m}\left(-A_{i}\right) .
\end{aligned}
$$

Here $-A_{i},-B_{j}$ means $A_{i}, B_{j}$ with opposite orientation.

If the surface $A_{1}$ is a component of $S_{1}$, by Claim 1 we have $A_{1} \cdot(a \times I) \neq 0$, then $A_{1}$ would separate $G \times 0$ from $G \times 1$, which contradicts to Claim 0 . 
Now $S_{2} \cap A_{1} \neq \emptyset$, a small isotopy will arrange that $\left|S_{1}^{\prime} \cap S_{2}^{\prime}\right|<\left|S_{1} \cap S_{2}\right|$. Moreover, $y\left(S_{1}^{\prime}\right)=y\left(S_{1}\right), y\left(S_{2}^{\prime}\right)=y\left(S_{2}\right)$. We want to show that $S_{1}^{\prime} \in \mathcal{S}_{p}(+C)$, $S_{2}^{\prime} \in \mathcal{S}_{q}(-C)$. Obviously, $\partial_{0} S_{1}^{\prime}=\partial_{0} S_{1}=C \times 0, \partial_{1} S_{1}^{\prime}=\partial_{1} S_{1}=-C \times 1$. Moreover, $S_{1}^{\prime} \cdot(a \times I)=S_{1} \cdot(a \times I)$ since $Q \cdot(a \times I)=0$. Thus $S_{1}^{\prime} \in \mathcal{S}_{p}(+C)$. Similarly, $S_{2}^{\prime} \in \mathcal{S}_{q}(-C)$. Therefore, we can replace $S_{1}, S_{2}$ with $S_{1}^{\prime}, S_{2}^{\prime}$, then continue our argument.

Now we are in a position to prove Lemma 3.6

Proof of Lemma 3.6. Suppose $y\left(\mathcal{S}_{p}(+C)\right)+y\left(\mathcal{S}_{q}(-C)\right) \leq(p+q) y(G)$. Let $S_{1}, S_{2}$ be as above, and suppose they satisfy Claims $1-3$. Define a function

$$
\varphi:(G \times I-P) \rightarrow \mathbb{Z}
$$

as follows. When $z \in G \times 0, \varphi(z)=0$. In general, given $z \in G \times I-P$, choose a path from $G \times 0$ to $z, \varphi$ is defined to be the algebraic intersection number of this path with $P$.

Any closed curve in $G \times I$ should have zero algebraic intersection number with any proper surface in $G \times \operatorname{int}(I)$, thus $\varphi$ is well-defined. Moreover, the value of $\varphi$ on $G \times 1$ is $p+q$.

Let $J_{i}$ be the closure of $\{x \in(G \times I-P) \mid \varphi(x)=i\}, P_{i}=J_{i-1} \cap J_{i}$. Thus $P=\sqcup_{i=1}^{m} P_{i}$ for some $m \geq p+q$, and $\cup_{k=0}^{i-1} J_{k}$ gives a homology between $G \times 0$ and $P_{i}$. $P$ is homologous to $(p+q) G$ in $G \times I, G \times 0$ is Thurston norm minimizing in $G \times I, y(P)=y\left(S_{1}\right)+y\left(S_{2}\right) \leq(p+q) y(G)$, so we must have $y\left(P_{i}\right)=y(G)$ for each $i$, and $m=p+q$ except possibly when $\chi(G)=0$. By Claims 1-3, we conclude that $m=p+q$ and $P_{i}$ is parallel to $G \times 0$ in $G \times I$.

Suppose $K \subset J_{r}$, then $J_{r}-\operatorname{int}(\operatorname{Nd}(K))$ is homeomorphic to $M_{2}$. Since $P$ is gotten by doing cut-and-paste to $S_{1}, S_{2}$, we can isotope $S_{1}$ so that $S_{1} \cap J_{i}$ consists of product annuli and disks. We denote $S_{1} \cap J_{i}$ by $C_{i} \times I$, where $C_{i}$ is the collection of some curves in $P_{i}$. Obviously, $\left[C_{i}\right]$ is homologous to $[C]$ in $\mathrm{H}_{2}(G, \partial G)$. Since $[C] \neq 0$, at least one component of $C_{i}$ is homologically nontrivial, which implies that $J_{r}-\operatorname{int}(\operatorname{Nd}(K))=M_{2}$ contains a nontrivial product disk or annulus, a contradiction.

Proof of Proposition 3.4 in Case 1. By Lemma 3.5. when $m$ is large there exist $S_{1} \in \mathcal{S}_{m}(+C), S_{2} \in \mathcal{S}_{m}(-C)$, such that they give taut decompositions of $M_{2}$. By the taut-extension property, $S_{1}, S_{2}$ also give taut decompositions of $G \times I$. Gabai's work in [2, Section 5] then implies that there exist two taut foliations $\mathscr{F}_{1}, \mathscr{F}_{2}$ of $G \times I$, such that $G \times\{0,1\}$ are compact leaves of the foliations, and $\mathscr{F}_{1}, \mathscr{F}_{2}$ are transverse to $\partial G \times I$.

Glue $G \times 0$ to $G \times 1$ by the identity, we get two taut foliations $\mathscr{F}_{1}^{\prime}, \mathscr{F}_{2}^{\prime}$ of $G \times S^{1}$. The two surfaces $S_{1}, S_{2}$ are glued to two surfaces $S_{1}^{\prime}, S_{2}^{\prime}$. We have $\chi\left(S_{i}^{\prime}\right)=\chi\left(S_{i}\right)-1=-y\left(S_{i}\right)$.

Let $e(\mathscr{F})$ be the Euler class of a foliation $\mathscr{F}$. As in the proof of 6 , Theorem 1.4], we have

$$
\begin{aligned}
& \chi\left(S_{1}^{\prime}\right)=\left\langle e\left(\mathscr{F}_{1}^{\prime}\right),\left[S_{1}^{\prime}\right]\right\rangle=\left\langle e\left(\mathscr{F}_{1}^{\prime}\right),\left[C \times S^{1}\right]\right\rangle+m \chi(G), \\
& \chi\left(S_{2}^{\prime}\right)=\left\langle e\left(\mathscr{F}_{2}^{\prime}\right),\left[S_{2}^{\prime}\right]\right\rangle=\left\langle e\left(\mathscr{F}_{2}^{\prime}\right),-\left[C \times S^{1}\right]\right\rangle+m \chi(G) .
\end{aligned}
$$


By Lemma 3.6] $\left\langle e\left(\mathscr{F}_{1}^{\prime}\right),\left[C \times S^{1}\right]\right\rangle+\left\langle e\left(\mathscr{F}_{2}^{\prime}\right),-\left[C \times S^{1}\right]\right\rangle\langle 0$, hence one summand on the left hand side is nonzero, which contradicts to [18, Corollary 1$]$.

Case 2. The curve $C$ is a circle or an arc with ends in the same component of $\partial G$.

If $C$ is an arc with ends in the same component of $\partial G$, we can connect the two ends by an arc in $\partial G$ to get a closed curve $\widehat{C}$. $\widehat{C}$ is homologous to $C$ in $\mathrm{H}_{1}(G, \partial G)$, so we can just work with $\widehat{C}$. The proof when $C$ is a circle is essentially the same as in Case 1 , it is even slightly simpler at some technical points. (For example, we can just work with the Thurston norm $x$, and do not need its modification $y$.) We will not give the details of the proof.

\section{Surgery on null-homotopic knots}

In this section, we are going to prove Theorem 1.1. The notation is as in the statement of Theorem 1.1.

Proposition 4.1. (Boileau-Wang, [1, Proposition 3.2]) Suppose $P$ is a compact 3-manifold, and $k$ is a null-homotopic knot in $P$. If $Q$ is obtained by Dehn surgery on $k$, then there is a proper degree-1 map from $Q$ to $P$. Let $k^{\prime} \subset Q$ be the core of the surgery, then the map can be chosen such that its restriction to $Q-\mathrm{Nd}\left(k^{\prime}\right)$ is a homeomorphism onto $P-\mathrm{Nd}(k)$.

Here a map $f: Q \rightarrow P$ is proper if $f^{-1}(\partial P)=\partial Q$. Note that in BoileauWang's original paper the result is stated for closed irreducible $3-$ manifolds, but the extra conditions are not necessary for the proof.

Lemma 4.2. Let $M=Y-\operatorname{int}(\operatorname{Nd}(L))$ be the exterior of the knot, then $M$ is irreducible.

Proof. We first consider the case that $X \neq S^{2} \times S^{1}$, thus $X$ is irreducible. If $S$ is an essential sphere in $M$, then $S$ bounds a 3 -ball $B$ in $X$, and $B \supset K$. Hence $S$ bounds a compact $3-$ manifold $B^{\prime}$ in $Y$, such that $L$ is a null-homotopic knot in $B^{\prime}$, and a nontrivial surgery on $L$ yields $B$.

By Proposition 4.1, there is a proper degree-1 map from the ball $B$ to $B^{\prime}$, hence $B^{\prime}$ is a homotopy 3 -cell (see [8, Lemma 15.12]). In other words, a nontrivial surgery on $K \subset B$ yields a homotopy 3 -cell. Now Property P [10] implies that $K$ is the unknot in $B$, so $L$ is the unknot in $B^{\prime}$, a contradiction.

Now consider the case that $X=S^{2} \times S^{1}$. If $S$ is a separating essential sphere in $M$, one can get contradiction by the same argument as before. Now suppose $S \subset M$ is a nonseparating sphere. Let $N$ (or $N^{\prime}$ ) be the compact manifold obtained by cutting $X$ (or $Y$ ) open along $S, \widehat{N}$ (or $\widehat{N^{\prime}}$ ) be the closed manifold obtained by capping off the 2 sphere boundary components of $N$ (or $N^{\prime}$ ) by balls. Now $L$ can be viewed as a nontrivial null-homotopic knot in $\widehat{N^{\prime}}$, such that a nontrivial surgery on $L$ yields $\widehat{N}=S^{3}$. Using Property $\mathrm{P}$, we can rule out this case as before. 
Lemma 4.3. $K$ can be isotoped to be disjoint from $F$.

Proof. By the homological restriction (1), there exists a unique element

$$
\theta \in \mathrm{H}_{2}(M, \partial M-T)
$$

whose image in $\mathrm{H}_{2}(X, \partial X)$ is $[F]$. Suppose $\left(F^{\prime}, \partial F^{\prime}\right) \subset(M, \partial M-T)$ is a taut surface in the homology class $\theta$. Since $L$ is null-homotopic in $Y$, by [12, Theorem A.21] $F^{\prime}$ is taut in $X$, hence $F^{\prime}$ is isotopic to the fibre $F$.

Lemma 4.4. $X \neq S^{2} \times S^{1}$.

Proof. Otherwise $M$ is reducible by Lemma 4.3, which violates Lemma 4.2 ,

Proposition 4.5 (Boileau-Wang). Let $\theta$ be the homology class in the proof of Lemma 4.3, $i_{Y}(\theta)$ is its image in $\mathrm{H}_{2}(Y, \partial Y)$. Then $Y$ is the connected sum of $Y^{*}$ and a homotopy 3-sphere, where $Y^{*}$ fibres over the circle, and the fibre of $Y^{*}$ represents the homology class $i_{Y}(\theta)$.

Proof. Let $p: \pi_{1}(Y) \rightarrow \mathbb{Z}$ be the homomorphism dual to $i_{Y}(\theta)$. By Proposition 4.1 and the proof of [1, Theorem 2.1], Ker $p$ is finitely generated. The result then follows from Stallings' Fibration Theorem [17].

Cut $X$ open along $F$, we get a product $F \times I$, thus $K$ is a knot in $F \times I$. Let $\left(M_{1}, \gamma_{1}\right),\left(M_{2}, \gamma_{2}\right), E, G$ be as in Section 3 . We can decompose $\left(M_{1}, \gamma_{1}\right)$ along a collection $\mathcal{C} \times I$ of non-separating product disks and annuli to get $\left(M_{2}, \gamma_{2}\right)$.

Proposition 4.6. If $M$ is $F_{T}$-atoroidal, then $M_{2}$ has the taut-extension property.

Proof. Suppose $S$ is a non-separating decomposition surface in the sutured manifold $\left(M_{2}, \gamma_{2}\right)$ such that $S \cap T=\emptyset$ and the decomposition

$$
\left(M_{2}, \gamma_{2}\right) \stackrel{S}{\rightsquigarrow}\left(M_{3}, \gamma_{3}\right)
$$

yields a taut sutured manifold,

As in the proof of Theorem 2.8, we can extend the taut decomposition sequence

$$
(M, \partial M)=\left(M_{0}, \gamma_{0}\right) \stackrel{F}{\rightsquigarrow}\left(M_{1}, \gamma_{1}\right) \stackrel{\mathcal{C} \times I}{\rightsquigarrow}\left(M_{2}, \gamma_{2}\right) \stackrel{S}{\rightsquigarrow}\left(M_{3}, \gamma_{3}\right)
$$

to a sequence

$$
\left(M_{0}, \gamma_{0}\right) \stackrel{F}{\rightsquigarrow}\left(M_{1}, \gamma_{1}\right) \stackrel{\mathcal{C} \times I}{\rightsquigarrow}\left(M_{2}, \gamma_{2}\right) \stackrel{S}{\rightsquigarrow}\left(M_{3}, \gamma_{3}\right) \stackrel{S_{4}}{\rightsquigarrow} \ldots \stackrel{S_{n}}{\rightsquigarrow}\left(M_{n}, \gamma_{n}\right)
$$

with the properties 1),2),3) there.

Fix a slope on $T$, then we can fill each $T \subset M_{i}$ along this slope by a solid torus to get the sequence

$$
\mathcal{N}:\left(N_{0}, \delta_{0}\right) \stackrel{F}{\rightsquigarrow}\left(N_{1}, \delta_{1}\right) \stackrel{\mathcal{C} \times I}{\rightsquigarrow} \ldots \stackrel{S_{n}}{\rightsquigarrow}\left(N_{n}, \delta_{n}\right) .
$$


As argued in Theorem 2.8, if the slope on $T$ is not the one that kills $s\left(\delta_{n}\right) \cap$ $\widehat{H}$ in $\pi_{1}(\widehat{H})$, then one can decompose $\left(N_{n}, \delta_{n}\right)$ along a $D^{2} \times$ point to get a product sutured manifold. Hence the above sequence $\mathcal{N}$ is extended to a sutured manifold hierarchy. Now we can apply [2, Theorem 5.1] to the sutured manifold hierarchy to obtain foliations as in the statement of Theorem 2.8

Since $L$ is null-homotopic in $Y$, the distinguished slope that kills $s\left(\delta_{n}\right)$ must be the meridian of $L$. As a result, the sequence $\mathcal{N}$ for the slope $\alpha$ is taut. In particular, the decomposition

$$
\left(G \times I=M_{2}(\alpha), \partial G \times I\right) \stackrel{S}{\rightsquigarrow}\left(M_{3}(\alpha), \delta_{3}\right)
$$

is taut.

Proof of Theorem 1.1 when $M$ is $F_{T}$-atoroidal. By Proposition 4.6] the condition in Proposition 3.4 is satisfied, so the only possibility of $G$ is that it is an annulus and

$$
i_{*}: \mathrm{H}_{1}(K ; \mathbb{Q}) \rightarrow \mathrm{H}_{1}(G ; \mathbb{Q})
$$

is an isomorphism. Hence $\partial(G \times I)$ is I-cobordant to $T=\partial \mathrm{Nd}(K)$. Since $M$ is $F_{T}$-atoroidal, $\partial(G \times I)$ is parallel to $T$, so $K$ is isotopic to the core of $G \times I$. This shows that $K$ can be isotoped to lie on $F$.

Let $\lambda$ be the slope on $T$ which is specified by $F . F$ is compressible in $M(\lambda)$, hence not taut there. Theorem 2.8 then asserts that $\beta=\lambda$.

Now consider the case when $M$ is not $F_{T}$-atoroidal, namely, there exists a torus $R \subset M_{1}$ which is I-cobordant to $T$ in $M_{1}$, but $R$ is not parallel to $T$.

Let us choose $R$ to be an "innermost" torus in $M_{1}$ which is I-cobordant to $T$ but not parallel to $T$. By Lemma 3.1, $R$ bounds a solid torus $U_{X}$ in $X$. Any torus in $M-\operatorname{int}\left(U_{X}\right)$ which is I-cobordant to $R$ is actually parallel to $R$.

Proof of Theorem 1.1 when the above torus $R$ is present. Let $K^{\prime}$ be the core of $U_{X}, U_{Y}$ be the manifold obtained from $U_{X}$ by $\beta$-surgery on $K$. By [5], one of the following cases must hold.

1) $U_{Y}=D^{2} \times S^{1}$. In this case $K$ is a braid in $U_{X}$, and $L$ is a braid in $U_{Y}$.

2) $U_{Y}=U^{\prime} \# W$, where $W$ is a closed 3-manifold and $1<\left|\mathrm{H}_{1}(W)\right|<\infty$.

3) $U_{Y}$ is irreducible and $\partial U_{Y}$ is incompressible.

In Case 1), let $L^{\prime}$ be the core of $U_{Y}$, then $L^{\prime}$ has finite order in $\pi_{1}(Y)$. By Proposition 4.5 $L^{\prime}$ is null-homotopic. $X$ can be viewed as obtained by a surgery on $L^{\prime}$. By the case we have proved, we have $\Delta\left(\alpha^{\prime}, \beta^{\prime}\right)=1$, where $\alpha^{\prime}$ is the meridian of $U_{X}, \beta^{\prime}$ is the meridian of $U_{Y}$.

Let $w, v$ be the winding numbers of $K, L$ in $U_{X}, U_{Y}$, then $\alpha^{\prime}$ cobounds a $w$-punctured disk $D_{X}$ with $w$ copies of $\alpha$ in $U_{X}-\operatorname{Nd}(K), \beta^{\prime}$ cobounds a $v$ punctured disk $D_{Y}$ with $v$ copies of $\beta$ in $U_{Y}-\mathrm{Nd}(L)$. Consider the intersection of $D_{X}, D_{Y}$, we get $\Delta\left(\alpha^{\prime}, \beta^{\prime}\right)=w v \Delta(\alpha, \beta)$, so $w=1$, which means that $K$ is the core of $U_{X}$, a contradiction to the assumption that $R$ is not parallel to $T$.

In Case 2), $Y$ would have a summand $W$, which contradicts to Proposition 4.5 
In Case 3), $L$ is a null-homotopic knot in $U_{Y}$, then by Proposition 4.1 and [8. Lemma 15.12], $\pi_{1}\left(U_{X}\right)=\mathbb{Z}$ surjects onto $\pi_{1}\left(U_{Y}\right)$, a contradiction to the incompressibility of $\partial U_{Y}$.

\section{Reducing the norm of fibred manifolds}

In this section, we are going to prove Theorem 1.4 The notation is as in Theorem 1.4 .

Lemma 5.1. $K$ can be isotoped in $X$ to be disjoint from $F$.

Proof. Otherwise, the Thurston norm of $\theta$ would be larger than $x(F)$. Let $\left(F^{\prime}, \partial F^{\prime}\right) \subset(M, \partial M-T)$ be a taut surface in the homology class $\theta$. By [3, Corollary 2.4], $F^{\prime}$ remains taut in at least one of $X$ and $Y$, which contradicts to the assumption that $x\left(F^{\prime}\right)>x(F)>x\left(i_{Y}(\theta)\right)$.

Cut $X$ open along $F$, we get a product $F \times I$, let $M_{1}, M_{2}, E, G$ be as in Section 3 ,

Proposition 5.2. If $M$ is $F_{T}$-atoroidal, then $M_{2}$ has the taut-decomposition property.

Proof. The proof is the same as Proposition 4.6 except that we use the fact that $x\left(i_{Y}(\theta)\right)<x(F)$ instead of the null-homotopicity of $L$.

Having Proposition 5.2 in hand, the proof of Theorem 1.4 when $M$ is $F_{T^{-}}$ atoroidal is the same as the proof of Theorem 1.1. Now let us consider the case $M$ is not $F_{T}$-atoroidal, namely, there exists a torus $R \subset M_{1}$ which is I-cobordant to $T$ in $M_{1}$, but $R$ is not parallel to $T$.

Let us choose $R$ to be an "innermost" torus in $M_{1}$ which is I-cobordant to $T$. By Lemma 3.1 $R$ bounds a solid torus $U_{X}$ in $F \times I$. Any torus in $M-\operatorname{int}\left(U_{X}\right)$ which is I-cobordant to $R$ is actually parallel to $R$.

Proof of Theorem 1.4 when the above torus $R$ is present. Let $K^{\prime}$ be the core of $U_{X} . U_{Y}$ is the manifold obtained from $U_{X}$ by $\beta$-surgery on $K$. Let $F_{1}$ be a norm minimizing surface in the homology class $i_{Y}(\theta) . R$ is I-cobordant to $T$, $\theta \in \mathrm{H}_{2}(M, \partial M-T)$, so $F_{1}$ can be isotoped to intersect $R$ in $2 n$ essential circles, such that the sum of these oriented circles is null-homologous in $R$. A standard argument enables us to surger $F_{1}$ to get a new surface $F_{2}$ in the same homology class, such that $\chi\left(F_{2}\right)=\chi\left(F_{1}\right)$, and $F_{2} \cap R=\emptyset$.

If the slope of $F_{1} \cap R$ does not bound a disk in $U_{Y}$, then $x\left(F_{2}\right)=x\left(F_{1}\right)$. Note that the components of $F_{2}$ in $U_{Y}$ are null-homologous, removing these components we get a surface $F_{3}$ in the same homology class, $x\left(F_{3}\right) \leq x\left(F_{2}\right)$. But $F_{3} \subset X$ is also a surface in the homology class of $[F]$ with $x\left(F_{3}\right) \leq x\left(F_{2}\right)=$ $x\left(F_{1}\right)<x(F)$, we get a contradiction.

Hence the slope of $F_{1} \cap R$ bounds a disk in $U_{Y}$, which means that $U_{Y}=$ $U^{\prime} \# W$, where $U^{\prime}$ is a solid torus and $W$ is a rational homology sphere by [5]. Let $\beta^{\prime}$ be the slope of $F_{1} \cap R, \alpha^{\prime}$ be the meridian of $U_{X}, M^{\prime}=X-\operatorname{int}\left(U_{X}\right)$. Let 
$Y^{\prime}$ be the manifold obtained from $M^{\prime}$ by $\beta^{\prime}$-filling, then $Y=Y^{\prime} \# W$. Since $W$ is a rational homology sphere, the Thurston norm of $Y^{\prime}$ is equal to the Thurston norm of $Y$. Apply the $F$-atoroidal case of Theorem 1.4 to $X, Y^{\prime}$, we know that $K^{\prime}$ can be isotoped to lie on $F, \beta^{\prime}$ is the slope specified by $F$.

Let $V=U_{X}-\operatorname{int}(\operatorname{Nd}(K))$. Suppose the winding number of $K$ in $U_{X}$ is $w>0$, then $\mathrm{H}_{1}(T)$ is generated by $w\left[\beta^{\prime}\right]$ and $\frac{1}{w}\left[\alpha^{\prime}\right]$ in $\mathrm{H}_{1}(V)$. Since $\beta^{\prime}$ bounds a disk $D$ in $U_{Y},\left[\beta^{\prime}\right]$ is an integer multiple of $[\beta]$ in $\mathrm{H}_{1}(V)$, namely,

$$
\left[\beta^{\prime}\right]=k\left(r w\left[\beta^{\prime}\right]+s \frac{1}{w}\left[\alpha^{\prime}\right]\right), \quad k, r, s \in \mathbb{Z} .
$$

One then deduces that $w=1$, so $V$ is a homology $T^{2} \times I$, hence $\mathrm{H}_{1}\left(U_{Y}\right) \cong \mathbb{Z}$ has no torsion. By [5], the only possibility is $U_{Y}=D^{2} \times S^{1}$, hence $K$ is a braid in $U_{X}$. But $w=1$, so $T$ is parallel to $R$, a contradiction.

\section{References}

[1] M Boileau, S Wang, Non-zero degree maps and surface bundles over $S^{1}$, J. Differential Geom. 43 (1996), no. 4, 789-806.

[2] D Gabai, Foliations and the topology of 3-manifolds, J. Differential Geom. 18 (1983), no. 3, 445-503.

[3] D Gabai, Foliations and the topology of 3-manifolds II, J. Differential Geom. 26 (1987), no. 3, 461-478.

[4] D Gabai, Foliations and the topology of 3-manifolds III, J. Differential Geom. 26 (1987), no. 3, 479-536.

[5] D Gabai, Surgery on knots in solid tori, Topology 28 (1989), no. 1, 1-6.

[6] P Ghiggini, Knot Floer homology detects genus-one fibred knots, to appear in Amer. J. Math., available at arXiv:math.GT/0603445.

[7] C Gordon, J Luecke, Knots are determined by their complements, J. Amer. Math. Soc. 2 (1989), no. 2, 371-415.

[8] J Hempel, 3-manifolds, Reprint of the 1976 original. AMS Chelsea Publishing, Providence, RI, 2004.

[9] R Kirby, Problems in low-dimensional topology, Geometric topology, Proceedings of the 1993 Georgia International Topology Conference held at the University of Georgia, Athens, GA, August 2-13, 1993. Edited by William H. Kazez. AMS/IP Studies in Advanced Mathematics, 2.2. American Mathematical Society, Providence, RI; International Press, Cambridge, MA, 1997.

[10] P Kronheimer, T Mrowka, Witten's conjecture and property P, Geom. Topol. 8 (2004), 295-310 (electronic). 
[11] M Lackenby, Surfaces, surgery and unknotting operations, Math. Ann. 308 (1997), no. 4, 615-632.

[12] M Lackenby, Dehn surgery on knots in 3-manifolds, J. Amer. Math. Soc. 10 (1997), no.4, 835-864.

[13] J Luecke, personal communication.

[14] Y Ni, Knot Floer homology detects fibred knots, Invent. Math. 170 (2007), no. 3, 577-608.

[15] M Scharlemann, Sutured manifolds and generalized Thurston norms, J. Differential Geom. 29 (1989), no. 3, 557-614.

[16] M Scharlemann, A Thompson, Link genus and the Conway moves, Comment. Math. Helv. 64 (1989), no. 4, 527-535.

[17] J Stallings, On fibering certain 3-manifolds, Topology of 3-manifolds and related topics (Proc. The Univ. of Georgia Institute, 1961) pp. 95-100, Prentice-Hall, Englewood Cliffs, New Jersey, 1962.

[18] W Thurston, A norm for the homology of 3-manifolds, Mem. Amer. Math. Soc. 59 (1986), no. 339, i-vi and 99-130. 